\title{
Isotropization of embedding Class I spacetime and anisotropic system generated by complexity factor in the framework of gravitational decoupling
}

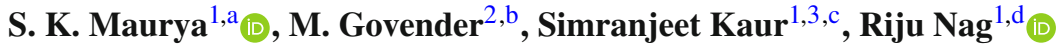 \\ ${ }^{1}$ Department of Mathematical and Physical Sciences, College of Arts and Sciences, University of Nizwa, Nizwa, Sultanate of Oman \\ ${ }^{2}$ Department of Mathematics, Durban University of Technology, Durban 4000, South Africa \\ ${ }^{3}$ Amity institute of Applied Sciences, Amity University, Uttar Pradesh, India
}

Received: 24 December 2021 / Accepted: 11 January 2022 / Published online: 2 February 2022

(C) The Author(s) 2022

\begin{abstract}
In this work, we present a hierarchical solutiongenerating technique employing the Minimum Gravitational Decoupling (MGD) Method and the generalized concept of Complexity as applied to Class I spacetime for bounded compact objects in classical general relativity. Starting off with an anisotropic seed solution described by Class I spacetime, we apply the MGD technique with the constraint that the effective anisotropy vanishes which leads to an isotropic model. In addition, we produce a second family of solutions in which the Complexity factor [Herrera (Phys Rev D 97:044010, 2018)] for the seed solution and its MGD counterpart are the same. We discuss the physical plausibility of both classes of solutions as candidates for physically realizable compact objects.
\end{abstract}

\section{Introduction}

Einstein's general theory of relativity (GTR) is the cornerstone of gravitation which has stood the test of time with the concordance of predictions and observations. GTR has cemented its place on the podium as a powerful mathematical formulation of gravity and this has been borne out in both astrophysical and cosmological fronts [1]. From the observed expansion of the universe, Cosmic Microwave Background Radiation, gravitational lensing, gravitational waves [3], deflection of starlight in the vicinity of massive gravitating bodies [2], perihelion precession of Mercury's orbit [4] through to the photographing of the shadow of a

\footnotetext{
a e-mail: sunil@unizwa.edu.om (corresponding author)

be-mail: megandhreng@dut.ac.za

${ }^{\mathrm{c}}$ e-mail: simmukr@gmail.com

de-mail: rijunag@gmail.com
}

black hole [5], GTR continues to reward us with a deeper understanding of gravitational phenomenology. With the success of GTR over the last century there are shortcomings that have also reared their unpretty heads such as the physics surrounding the initial Big Bang singularity, observed acceleration of the universe, end-states of continued gravitational collapse, amongst others. In order to explain some of these shortfalls and/or observations, researchers had to appeal to conjuring up exotic matter fields such as dark matter, dark energy, Skyrme fluids, Chameleons, to name a few [6]. The $\Lambda$-CDM cosmological model seems to be a strong candidate which can account for present-day observations via the Planck Collaboration of Age, mass density and the Hubble constant within experimental error. With the next generation of detectors and satellites will require fine-tuning of existing cosmological models or a complete overhaul of current mathematical formulations [7].

On the astrophysical front, GTR has provided us with a plethora of exact solutions describing stellar objects. Since the discovery of the exterior Schwarzschild solution which describes the exterior gravitational field of a static, bounded configuration in GTR in 1916 [8]. Relativistic astrophysics has grown in stature describing gravitational collapse, transport phenomena in stellar objects, stability of equilibrium configurations, observed luminosities and temperature profiles of compact objects amongst others [9-11]. In particular, the Cosmic Censorship Hypothesis [12] has attracted the attention of researchers since the pioneering work of Oppenheimer and Snyder [13] on continued gravitational collapse of an idealised dust sphere. This area has grown in leaps and bounds with more realistic scenarios of gravitational collapse being probed. Physics surrounding the final outcome of gravitational collapse, particularly at ultra-high densities of the order of $10^{18} \mathrm{~g} / \mathrm{cm}^{3}$ required modifications of GTR. Besides 
the search for a consistent and complete quantum theory of gravity, extensions and modifications of GTR have provided us with interesting new results which provide an increased parameter space to account for observations.

Alternative theories to GTR include Brans-Dicke (BD) scalar-tensor theory of gravity [14] which embodies Mach's principle. In this formulation the non-minimally coupled scalar field mimics the spacetime-varying gravitational "constant". While there are other scalar theories of gravity, the Brans-Dicke theory continues to be a preferred choice, particularly amongst cosmologists. BD formulation of gravity has been utilised to inflation in the early universe and the current accelerated epoch of the universe without the need for exotic matter fields such as dark energy. A recent investigation presented a solution of the Einstein-Maxwell field equations in the presence of a massive scalar field in the Brans-Dicke (BD) gravity which describes charged anisotropic strange stars. A novel finding in this work showed that the electric field and scalar field which have completely different sources coupled to impact physical features such as mass-radius relation and surface redshifts of compact objects.

Further extensions and modifications to classical GTR include the $f(R)$ gravity framework proposed by Buchdahl (1959). In this formalism the action is quadratic in the Ricci scalar. One of the pathologies of the $f(R)$ gravity is the prediction of ghost fields associated with higher order derivatives. The $f(R)$ gravity theory was utilised by Starobinsky to produce a family of cosmological solutions which differ from the current $\Lambda$-CDM model but can account for observations on cosmological, Solar system and laboratory scales [15]. A natural extension of GTR to higher dimensions is the so-called Lovelock gravity. GTR is indistinguishable from Lovelock gravity in $D=3$ and 4 dimensions since higher curvature contributions masquerade as a total derivative and do not affect the dynamics $[16,17]$. The so-called EinsteinGauss-Bonnet (EGB) is a special case of a second-order Lovelock polynomial and holds for dimensions $D=5$ and 6. Further extensions to GTR include $f(R, T)$, Rastall and $f(T)$ theories $[18,19]$. These theories have been successfully utilised to model compact objects such as neutron stars, pulsars and strange stars.

The role of anisotropy in self-gravitating objects has attracted a wide spectrum of interest amongst researchers, especially over the past decade. The study of radiating stars in the presence of heat dissipation and pressure anisotropy has provided us with a myriad of interesting results regarding stability, causality, thermodynamics and the end-state of collapse. It was shown that the time of formation of the horizon is advanced when the principal stresses are unequal within the stellar fluid [20]. In addition, pressure anisotropy leads to higher core temperatures within the collapsing configuration. Gravitational decoupling (GD) and its articulation via minimal geometric deformation (MGD) method [21] and its generalisation, the complete geometric deformation (CGD) [22] have enabled researchers to produce anisotropic models from known isotropic solutions. MGD and CDG approaches have led to an exponential growth of stellar models in both GTR and modified gravity theories. A recent investigation demonstrated that contributions from the decoupling parameter and the EGB constant predict neutron stars with masses greater than $M=2 M_{\odot}$ without invoking exotic matter fields [23]. Some more recent works in different contexts using MGD and CGD can be found in the following Refs.[2453].

Recently there has been heightened interest in the concept of complexity in self-gravitating systems, an idea largely explored by Herrera and co-workers for static and dynamical systems [54-56]. They proposed a definition of the so-called complexity factor arising from the orthogonal splitting of the Riemann tensor which gives rise to scalar structures. These scalars inherently link the local anisotropy of the transverse and radial stresses and density inhomogeneity to the Tolman mass for a static, bounded stellar configuration. The simplest static, self-gravitating system with a vanishing complexity factor is the one featuring isotropic pressure and homogeneous density. It follows that the vanishing of the complexity factor implies either, homogeneous energy density and pressure isotropy, or inhomogeneous energy density and pressure anisotropy. In order to close the system of equations governing the gravitational behaviour of a static star, many of the recent studies have invoked the condition of vanishing complexity factor as an additional constraint on the system. As mentioned earlier gravitational decoupling method facilitates the anisotropisation of isotropic seed solutions describing bounded configurations in GTR and modified gravity theories. Gravitational decoupling and the vanishing of the complexity factor have been widely exploited to produce compact stellar models describing neutron stars, pulsars and strange stars [57-59].

The paper is organized as follows: In Sect. 1 we provide an over-arching introduction of gravitational theory and the context of the problem under study. In Sect. 2, we present the gravitationally decoupled Einstein field equations for two sources which are divided into two Sects. 2.1 and 2.2. In Sect. 3 , we discuss the complexity factors for the gravitationally decoupled systems. The new gravitationally decoupled Class I solutions have been presented in Sect. 4 which contain two Sects. 4.1 and 4.2. In Sect. 4.1, we generate a Class I MGD solution by using isotropization technique while new version of Class I anisotropic solution using two systems with the same complexity factor is given in Sect. 4.2. The physical properties of the solutions are discussed in Sect. 5. The last Sect. 6 contains the concluding remarks of the article. 


\section{Gravitationally decoupled Einstein field equation (EFE) generated by two sources}

\subsection{Field equations for gravitationally decoupled system}

In this section, we present a concise review of gravitationally decoupled Einstein field equations for two independent sources with the relativistic units $G=c=1$ as,

$$
G_{i j}=R_{i j}-\frac{1}{2} g_{i j} R=-8 \pi \hat{T}_{i j}
$$

with,

$$
\hat{T}_{i j}=T_{i j}+\beta \Theta_{i j}
$$

As usual the Ricci tensor is symbolized by $R_{i j}$, where $R$ reflects contracted Ricci scalar and $\beta$ indicates the decoupling constant in the field equations. The energy-momentum tensor is symbolized by $T_{i j}$, and the source $\theta_{i j}$ may incorporate new fields such as scalar, vector, and tensor fields. Since the Bianchi identity is satisfied by the Einstein tensor $\left(G_{i j}\right)$, then the effective energy-momentum tensor $\hat{T}_{i j}$ must be maintained,

$$
\nabla_{i} \hat{T}^{i j}=0
$$

For expressing the space-time of the interior region for the stellar system, the following static spherically symmetric line element is being used,

$d s^{2}=-e^{\lambda(r)} d r^{2}-r^{2}\left(d \theta^{2}+\sin ^{2} \theta d \phi^{2}\right)+e^{v(r)} d t^{2}$,

where $v$ and $\lambda$ are only radially dependent metric potentials. We suppose that the internal structure of the self-gravitating system described by energy-momentum tensor $T_{i j}$ represents an anisotropic matter distribution, then

$$
T_{i j}=\left(\rho+p_{r}\right) u_{i} u_{j}+p_{t} g_{i j}+\left(p_{r}-p_{t}\right) \chi_{i} \chi_{j},
$$

The radial and tangential pressures are indicated by $p_{r}$ and $p_{t}$, correspondingly, whereas the energy density of matter is denoted by $\rho$. In addition, $u^{i}$ is a contravariant 4-velocity, and $\chi^{i}=\sqrt{1 / g_{r r}} \delta_{1}^{i}$ is a unit space-like vector in the radial direction. The components of the effective energy-momentum tensor $\hat{T}_{i j}$ are taken as,

$\hat{T}_{0}^{0}=\epsilon, \quad \hat{T}_{1}^{1}=-P_{r}, \quad$ and $\hat{T}_{2}^{2}=-P_{\perp}$.

Using Eqs. (4)-(6), the Einstein field equation (EFE) (1) yields the following differential equations,

$$
\begin{aligned}
& 8 \pi \epsilon=\frac{1}{r^{2}}-e^{-\lambda}\left(\frac{1}{r^{2}}-\frac{\lambda^{\prime}}{r}\right), \\
& 8 \pi P_{r}=-\frac{1}{r^{2}}+e^{-\lambda}\left(\frac{1}{r^{2}}+\frac{v^{\prime}}{r}\right), \\
& 8 \pi P_{\perp}=\frac{e^{-\lambda}}{4}\left(2 v^{\prime \prime}+v^{\prime 2}-\lambda^{\prime} v^{\prime}+2 \frac{v^{\prime}-\lambda^{\prime}}{r}\right),
\end{aligned}
$$

where,

$$
\epsilon=\rho+\beta \Theta_{0}^{0}, \quad P_{r}=p_{r}-\beta \Theta_{1}^{1}, \text { and } P_{\perp}=p_{t}-\beta \Theta_{2}^{2} .
$$

and the conservation equation of system (7)-(9) is,

$\left(P_{r}\right)^{\prime}+\frac{\nu^{\prime}}{2}\left(\epsilon+P_{r}\right)-\frac{2}{r}\left(P_{\perp}-P_{r}\right)=0$,

However, the explicit form of the conservation equation (11) can be rewritten by using Eq. (10) as

$$
\begin{aligned}
& p_{r}^{\prime}+\frac{\nu^{\prime}}{2}\left(\rho+p_{r}\right)+\frac{2\left(p_{r}-p_{t}\right)}{r} \\
& -\beta\left[\left(\Theta_{1}^{1}\right)^{\prime}+\frac{\nu^{\prime}}{2}\left(\Theta_{0}^{0}-\Theta_{1}^{1}\right)+\frac{2}{r}\left(\Theta_{1}^{1}-\Theta_{2}^{2}\right)\right] .
\end{aligned}
$$

Now we defined $\theta$-components in form of new variables as [60],

$\rho^{\Theta}=\beta \Theta_{0}^{0}, \quad p_{r}^{\Theta}=-\beta \Theta_{1}^{1}$, and $p_{t}^{\Theta}=-\beta \Theta_{2}^{2}$.

The effective anisotropy can be expressed as,

$\hat{\Pi}=P_{\perp}-P_{r}=\Pi+\Pi_{\Theta}$,

where,

$$
\Pi=p_{t}-p_{r} \text { and } \Pi_{\Theta}=p_{t}^{\Theta}-p_{r}^{\Theta}=\beta\left(\Theta_{1}^{1}-\Theta_{2}^{2}\right)
$$

The second source $\Theta_{i j}$ generates the anisotropy $\Pi_{\Theta}$, however the Misner-Sharp mass function $m(r)$ for the effective system may be computed using the formula,

$$
\begin{aligned}
m(r) & =\frac{r}{2}\left[1-e^{-\lambda(r)}\right]=4 \pi \int_{0}^{r} x^{2} \epsilon(x) d x \\
& =\underbrace{4 \pi \int_{0}^{r} x^{2} \rho(x) d x}_{m_{G R}}+\underbrace{4 \pi \beta \int_{0}^{r} x^{2} \Theta_{0}^{0}(x) d x}_{m_{\Theta}} .
\end{aligned}
$$

The mass functions relating to matter distribution $T_{i j}$ and $\Theta_{i j}$ are represented by $m_{G R}$ and $m_{\Theta}$, respectively.

Many years ago, Tolman [61] introduced another definition for the energy content inside a fluid sphere. Using the definition in [61], we can calculate the Tolman mass function $m_{T}$ for the spherically symmetric static spacetime (4) under the energy-momentum tensor $\hat{T}_{i j}$,

$m_{T}=4 \pi \int_{0}^{r} x^{2} e^{(\lambda+\nu) / 2}\left(\rho+P_{r}+2 P_{\perp}\right) d x$,

The formula above was suggested to determine the amount of energy contained within a fluid sphere of radius $r$. Furthermore, using the field equations (7)-(9) under the spacetime (4), the Tolman mass function $m_{T}$ maybe interpreted as,

$m_{T}=\frac{r^{2} v^{\prime}}{2} e^{(\nu-\lambda) / 2}$, 
The gravitational acceleration of a test particle instantaneously at rest in a static gravitational field can be described by the formula [54],

$a=\frac{\nu^{\prime} e^{-\lambda}}{2}=\frac{e^{-\nu / 2} m_{T}}{r^{2}}$.

2.2 The field equations for the systems $T_{i j}$ and $\Theta_{i j}$ generated by MGD approach

In this section, we will find the field equations for the two systems $T_{i j}$ and $\Theta_{i j}$ by using the minimal geometric deformation (MGD) approach. To do this, we apply the transformation over the metric functions $e^{\lambda}$ and $e^{\nu}$, which is as follows [22],

$$
\begin{aligned}
& \xi(r) \mapsto v(r)=\xi(r)+\beta h(r), \\
& \mu(r) \mapsto e^{-\lambda(r)}=\mu(r)+\beta f(r) .
\end{aligned}
$$

The geometric deformation functions for the radial and temporal metric components are denoted by $f(r)$ and $h(r)$, respectively. We need to set $f(r) \neq 0$ and $h(r)=0$ since we're dealing with the minimal geometric approach here. The transformations (20) and (21), then allow us to separate the field equations (7)-(9) into the following two sets: (i) the Einstein field equations for the energy-momentum tensor $T_{i j}$ (same as for setting $\beta=0$ ) as

$$
\begin{aligned}
& 8 \pi \rho=\frac{1-\mu}{r^{2}}-\frac{\mu^{\prime}}{r}, \\
& 8 \pi p_{r}=\frac{\mu-1}{r^{2}}-\frac{\mu \xi^{\prime}}{r}, \\
& 8 \pi p_{t}=\mu\left(\frac{\xi^{\prime \prime}}{2}+\frac{\xi^{\prime 2}}{4}+\frac{\xi^{\prime}}{2 r}\right)+\left(\frac{\xi^{\prime} \mu^{\prime}}{4}+\frac{\mu^{\prime}}{2 r}\right),
\end{aligned}
$$

with the equation of conservation as,

$\left(p_{r}\right)^{\prime}+\frac{\xi^{\prime}}{2}\left(\rho+p_{r}\right)=\frac{2 \Pi}{r}$.

and the following spacetime could be used to explain the solution of the system (22)-(24),

$d s^{2}=-\mu d r^{2}-r^{2}\left(d \theta^{2}+\sin ^{2} \theta d \phi^{2}\right)+e^{\xi} d t^{2}$,

with

$\mu=1-\frac{2 m_{G R}}{r}=4 \pi \int_{0}^{r} x^{2} \rho(x) d x$. (ii) Turning on $\beta$ now determines the second system of equations for the extra source $\Theta_{i j}$ as,

$$
\begin{aligned}
& 8 \pi \rho^{\Theta}=-\beta\left(\frac{f^{\prime}}{r}+\frac{f}{r^{2}}\right), \\
& 8 \pi p_{r}^{\Theta}=\beta f\left(\frac{\xi^{\prime}}{r}+\frac{1}{r^{2}}\right), \\
& 8 \pi p_{t}^{\Theta}=\frac{\beta f}{2}\left(\xi^{\prime \prime}+\frac{\xi^{\prime 2}}{2}+\frac{\xi^{\prime}}{r}\right)-\frac{\beta f^{\prime}}{2}\left(\frac{\xi^{\prime}}{2}+\frac{1}{r}\right) .
\end{aligned}
$$

whose conservation equation is as follows,

$$
\frac{\xi^{\prime}}{2}\left(\rho^{\Theta}+p_{r}^{\Theta}\right)+\left(p_{r}^{\Theta}\right)^{\prime}=\frac{2 \Pi_{\Theta}}{r} .
$$

\section{Complexity formula by gravitational decoupling}

Recently, Herrera [54] established the concept of the complexity factor in static and spherically symmetric selfgravitating systems, which is a scalar function represented by $Y_{T F}$ and can be calculated using anisotropy $\Pi$ and energy density gradient $\rho^{\prime}$. In this continuation, Herrera and his collaborators later developed this complexity in the setting of dissipative self-gravitating fluid distributions with dynamical spherically symmetric dissipation. In the present case, we represent $\hat{Y}_{T F}$ as a complexity factor for the spherically symmetric static self-gravitating systems (7)-(9), which can be written according to the Herrera definition as,

$\hat{Y}_{T F}=8 \pi \hat{\Pi}-\frac{4 \pi}{r^{3}} \int_{0}^{r} x^{3} \epsilon^{\prime}(x) d x$.

Herrera mentioned that the complexity factor $\hat{Y}_{T F}$ demonstrates the influence of local anisotropy of pressure and density inhomogeneity on the Tolman mass $\left(m_{T}\right)$, or how the Tolman mass is affected by the two parameters listed in $\hat{Y}_{T F}$. To show the effect of $\hat{Y}_{T F}$ on the Tolman $m_{T}$, we formulate Eq. (17) in terms of complexity factor as follows:

$m_{T}=M_{T}\left(\frac{r}{R}\right)^{2}+r^{3} \int_{r}^{R} \frac{e^{(v+\lambda) / 2}}{x} Y_{T F} d x$.

Here, the total Tolman mass of the fluid sphere of radius $R$ is denoted by $M_{T}$ (see the Ref. [54] for more details about the complexity). Recently, Casadio and his collaborators discussed the complexity factor in the context of MGD approach, and they found that the complexity factor satisfies the additive property. The complexity factor for gravitationally decoupled systems given by Eq. (32) can be presented as,

$$
\begin{aligned}
\hat{Y}_{T F}= & 8 \pi \Pi-\frac{4 \pi}{r^{3}} \int_{0}^{r} x^{3} \rho^{\prime}(x) d x \\
& +8 \pi \beta \Pi_{\Theta}-\frac{4 \pi \beta}{r^{3}} \int_{0}^{r} x^{3}\left[\Theta_{0}^{0}(x)\right]^{\prime} d x,
\end{aligned}
$$




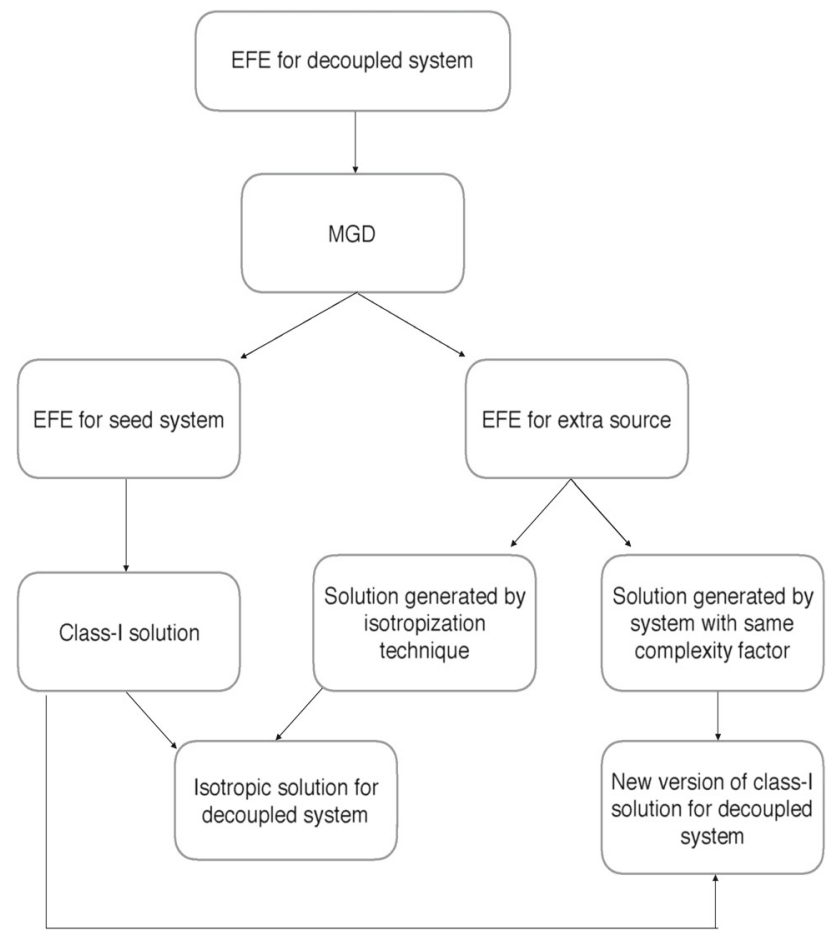

Fig. 1 The flow chart shows the systematic approach adopted for generating the solution in the context of MGD

The above Eq. (34) can be now written as,

$$
\hat{Y}_{T F}=Y_{T F}+Y_{T F}^{\Theta} \text {. }
$$

which is the sum of two existing complexity factors induced by the sources $T_{i j}$ and $\Theta_{i j}$. Then, $Y_{T F}$ will represent the complexity factor for the system (22)-(24), whereas $Y_{T F}^{\Theta}$ describes the complexity factor for (28)-(30).

\section{New gravitationally decoupled solutions by MGD approach}

In this section, we are going to discuss in particular two scenarios,

(A) the solution for gravitationally decoupled system (5)(8) generated by isotropization technique and corresponding complexity factor,

(B) some new minimally deformed solutions for the system (5)-(8) generated by two systems having the same complexity factor.

\subsection{Solution generated by isotropization technique}

In this section, we will use Casadio et al. [60] systematic technique to isotropize the decoupled system (7)-(9) under the
MGD scenario. As a result of introducing the source $\Theta_{i j}$, we may transform an anisotropic system (22)-(24) with $\Pi \neq 0$ provided by $T_{i j}$ into an isotropic system (7)-(11) generated by $\hat{T}_{i j}$ with $\hat{\Pi}=0$. This transformation can be managed by setting the decoupling parameter $\beta=0$ and $\beta=1$, which represent the anisotropic system (22)-(24) and isotropic system (7)-(9), respectively. Then the isotropization is done by setting $\beta=1$, for which $\hat{\Pi}=0$ gives,

$\Pi=-\Pi_{\Theta} \Longrightarrow \Pi=-\left(\Theta_{1}^{1}-\Theta_{2}^{2}\right)$.

Now we obtain the following non-linear differential equation by substituting of Eqs. (29) and (30) into Eq. (36) as

$f^{\prime}\left(2+\xi^{\prime} r\right)-f\left(4+2 \xi^{\prime} r-2 \xi^{\prime \prime} r^{2}-\xi^{\prime 2} r^{2}\right)+4 r^{2} \Pi=0$.

As we know that in general the solution of the $\theta$-sector in the gravitational decoupling is reliant on the solution of the first system. Therefore, as a result, we must solve the first system initially. In order to solve the first system, we employ the Karmarkar condition that represents an embedding Class I solution which can be derived from the Riemann curvature tensor $R_{\mu \nu \gamma \delta}$. In this connection, Eisenhart proposed a significant and vital criterion for the embedding Class-I solution [62], which states that if there exists a second-order symmetric tensor $b_{i j}=b_{j i}$ for which symmetric tensor $b_{\mu \nu}$ satisfies the following conditions:

i. Gauss's equation

$\mathcal{R}_{\mu \nu \gamma \delta}=\epsilon\left(b_{\mu \alpha} b_{\nu \beta}-b_{\mu \beta} b_{\nu \alpha}\right)$,

ii. Codazzi's equation

$\nabla_{\alpha} b_{\mu \nu}-\nabla_{\nu} b_{\mu \alpha}=0$

where $\epsilon= \pm 1$ whenever the normal to the manifold is spacelike $(+1)$ or time-like $(-1)$. The Karmarkar condition is derived by using Eqs. (38) and (39) through some mathematical computation as

$$
\frac{R_{1212} R_{3030}+R_{1220} R_{1330}}{R_{1010} R_{2323}}=1,
$$

Here, $R_{2323} \neq 0$. Then, considering the spherically symmetric spacetime (25), the Eq. (40) results in the following differential equation,

$\frac{2 \xi^{\prime \prime}}{\xi^{\prime}}+\xi^{\prime}=\frac{-\mu^{\prime}}{\mu(1-\mu)}$,

with $\mu \neq 1$. After integration of Eq. (41), we get

$$
\begin{aligned}
& \xi(r)=2 \ln \left[B\left(1+A \int \sqrt{(1-\mu) / \mu} d r\right)\right] \text { or } \\
& \mu(r)=\left(1+\frac{K e^{\xi}}{4} \xi^{\prime 2}\right) .
\end{aligned}
$$


The Class I condition (42) provides the relation between two metric potentials, which can be solved if either of the potentials is known. Therefore, we assume a well-behaved metric potential $\xi=2 A r^{2}+\ln B$ to solve the Class I condition (42), where $A$ and $B$ are constants. By substituting the said metric potential in condition (42) and integrating, we obtain the following Class I solution which describes a spacetime

$d s^{2}=-\left(1+D A r^{2} e^{2 A r^{2}}\right) d r^{2}-r^{2} d \Omega_{2}^{2}+B e^{2 A r^{2}} d t^{2}$.

The constant parameters $A, B$, and $D$ will be determined by using the boundary conditions. Using $\xi$ and $\mu$ from the above Class I spacetime, the system (22)-(24) provides the energy density and pressure's expressions for the energymomentum tensor $T_{i j}$ as,

$p_{r}=-\frac{A\left(-4+D e^{2 A r^{2}}\right)}{1+A D e^{2 A r^{2}} r^{2}}$,

$p_{t}=\frac{A\left(4-D e^{2 A r^{2}}+4 A r^{2}\right)}{\left(1+A D e^{2 A r^{2}} r^{2}\right)^{2}}$,

$\rho=\frac{A D e^{2 A r^{2}}\left(3+A\left(4+D e^{2 A r^{2}}\right) r^{2}\right)}{\left(1+A D e^{2 A r^{2}} r^{2}\right)^{2}}$.

Using the Eqs. (44)-(46) into Eq. (36) and integrating, we find the deformation function $f(r)$ as

$$
\begin{aligned}
f(r)= & e^{-2 A r^{2}} r^{2}\left(F-\frac{2 A \text { ExpIntegralEi }\left[1+2 A r^{2}\right]}{e}\right. \\
& \left.+\frac{A D e^{4 A r^{2}}}{\left(1+A D e^{2 A r^{2}} r^{2}\right)}\right)
\end{aligned}
$$

where, $F$ is a constant of integration. Furthermore, the suitable boundary conditions for the solution (44)-(46) are determined by the well-known Israel-Darmois junction conditions. These conditions are called the continuity of first and second fundamental across the boundary of the star, respectively. In order to invoke the junction conditions we must smoothly match the interior Class I spacetime (43) with exterior vacuum spacetime described by the exterior Schwarzschild solution. The matching yields the vanishing of radial pressure at the surface $r=R$ and fixes the arbitrary constants in the solution. The explicit form of above matching conditions can be written as,

$$
\begin{aligned}
& 1-\frac{2 M_{s}}{R}=e^{\xi(R)}, \\
& 1-\frac{2 M_{s}}{R}=\mu(R), \\
& p_{r}(R)=0,
\end{aligned}
$$

where $m_{G R}(R)=M_{S}$ represents the total mass of the object with radius $R$ for the Class I spacetime (43) described by

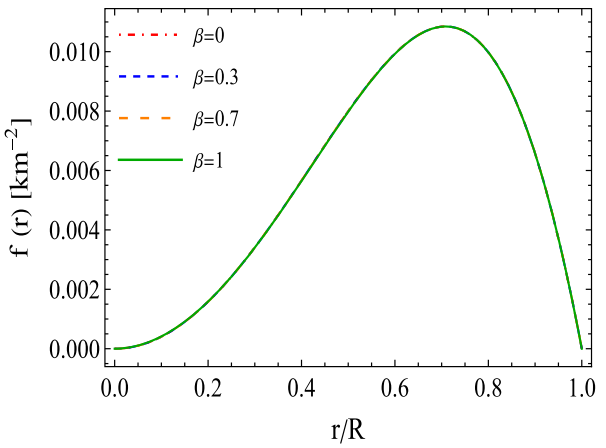

Fig. 2 The behavior of the deformation function versus radial coordinate $r / R$ for the solution 4.1 corresponding to the case $\hat{\Pi}=0$

source $T_{i j}$. The conditions (48)-(50) determine the constants $A, B$, and $D$ as

$$
\begin{aligned}
& A=-\frac{M_{s}}{2\left(M_{s}-R\right) R^{2}}, \\
& B=\frac{-4 M_{s}^{2}+5 M_{s} R-R^{2}}{\left(2 M_{s}-R\right) R}, \\
& D=4 e^{\frac{M_{s}}{2 M_{s}-R}} .
\end{aligned}
$$

Now the minimally deformed Class I solution for the system (7)-(9) can be given by the following spacetime,

$$
\begin{aligned}
d s^{2}= & -\frac{\left(1+D A r^{2} e^{2 A r^{2}}\right)}{1+\beta\left(1+D A r^{2} e^{2 A r^{2}}\right) f(r)} d r^{2}-r^{2} d \Omega_{2}^{2} \\
& +B e^{2 A r^{2}} d t^{2} .
\end{aligned}
$$

whose matter variables such as the effective pressures and energy density are given,

$$
\begin{aligned}
& P_{r}(r, \beta)=\beta \Psi_{1}(r)\left(4 A r^{2}+1\right)-\frac{A\left(D e^{2 A r^{2}}-4\right)}{1+A D e^{2 A r^{2}} r^{2}} \\
& P_{\perp}(r, \beta) \\
& =\frac{\beta\left[\Psi_{2}(r)+2 A \Psi_{2}(r) r^{2}+8 A r^{2} \Psi_{1}(r)\left(1+A r^{2}\right)\right]}{2} \\
& +\frac{A\left(4-D e^{2 A r^{2}}+4 A r^{2}\right)}{\left(1+A D e^{2 A r^{2}} r^{2}\right)^{2}}, \\
& \epsilon(r, \beta)=\frac{A D\left[3+A\left(4+D e^{2 A r^{2}}\right) r^{2}\right]}{e^{-2 A r^{2}}\left(1+A D e^{2 A r^{2}} r^{2}\right)^{2}} \\
& \quad-\beta\left[\Psi_{1}(r)+\Psi_{2}(r)\right] .
\end{aligned}
$$

where, the functions $\Psi_{1}(r)$ and $\Psi_{2}(r)$ are mentioned in the Appendix. However, the effective anisotropy $\hat{\Pi}$ is calculated as,

$\hat{\Pi}=\frac{A^{2}(1-\beta)\left(D e^{2 A r^{2}}-2\right)^{2} r^{2}}{\left(1+A D e^{2 A r^{2}} r^{2}\right)^{2}}$ 
where $m(R)=M$ is the total mass of the minimally deformed Class I compact object of radius $R$ generated by the solution (54)-(58). From the above Eq. (58), it can be clearly observed that $\hat{\Pi}$ becomes zero at $\beta=1$, which represents the system (5)-(8) to be an anisotropic system. On the other hand, the matching conditions (48)-(50) for new solution (54)-(58),

$$
\begin{aligned}
e^{-\lambda(R)} & =\frac{1}{\left(1+D A r^{2} e^{2 A r^{2}}\right)}+\beta f(R)=1-\frac{2 M}{R}, \\
e^{\nu(R)} & =B e^{2 A R^{2}}=1-\frac{2 M}{R}, \\
P_{r}(R) & =0
\end{aligned}
$$

determine the constant $B$, total mass $M$, and integration constant $F$ as

$$
\begin{aligned}
B= & \ln \left[\frac{1}{1+A D e^{2 A R^{2}} R^{2}}+\beta f(R)\right]-2 A R^{2} \\
M= & M_{s}-\frac{\beta R}{2} f(R), \\
F= & \frac{1}{\beta e\left(4 A R^{2}+1\right)\left(A D R^{2} e^{\left.2 A R^{2}+1\right)}\right.}\left[A \left\{2 \beta \left(4 A R^{2}\right.\right.\right. \\
& +1)\left(A D R^{2} e^{2 A R^{2}}+1\right) \text { ExpIntegralEi }\left[2 A R^{2}+1\right] \\
& \left.\left.-\left(4+D e^{2 A R^{2}} e^{2 A R^{2}+1}\left(4 A \beta R^{2}+\beta-1\right)\right)\right\}\right] .
\end{aligned}
$$

Furthermore, we will discuss the complexity factor for the above minimally deformed Class I solution (54)-(58) in the next section.

\subsubsection{Complexity factor generated by minimally deformed Class I solution (54)-(58):}

As mentioned earlier, the complexity factor of the systems (7)-(9) for energy-momentum tensor $\hat{T}_{i j}$ is

$\hat{Y}_{T F}=8 \pi \hat{\Pi}-\frac{4 \pi}{r^{3}} \int_{0}^{r} x^{3} \epsilon^{\prime}(x) d x$,

Since the solution (54)-(57) for system (5)-(9) is determined by taking $\hat{\Pi}=0$, then the Eq. (65) gives

$\hat{Y}_{T F}=-\frac{4 \pi}{r^{3}} \int_{0}^{r} x^{3} \epsilon^{\prime}(x) d x$,

Inserting of Eq. (5) into Eq. (66) gives

$\hat{Y}_{T F}=\frac{1}{r^{2}}-\frac{e^{-\lambda}}{r^{2}}-\frac{\lambda^{\prime} e^{-\lambda}}{2 r}$, and then we find the complexity factor $\hat{Y}_{T F}$ by using the solution(54) as,

$$
\begin{aligned}
& \hat{Y}_{T F} \\
& =\frac{A e^{-1-2 A r^{2}} r^{2}}{\left(1+2 A r^{2}\right)\left(1+A D e^{2 A r^{2}} r^{2}\right)^{2}}\left[-e\left\{A D e^{4 A r^{2}}(2\right.\right. \\
& \left.-D e^{2 A r^{2}}\right)\left(1+2 A r^{2}\right)+\beta\left(2 F\left(1+A D e^{2 A r^{2}} r^{2}\right)^{2}(1\right. \\
& \left.+2 A r^{2}\right)+A e^{2 A r^{2}}\left[4+2 D e^{2 A r^{2}}\left(-1+2 A r^{2}\right)+D^{2}(1\right. \\
& \left.\left.\left.\times e^{4 A r^{2}}\left(1+2 A r^{2}+4 A^{2} r^{4}\right)\right]\right)\right\}+4 A \beta\left(1+2 A r^{2}\right)(1 \\
& \left.\left.+A D e^{2 A r^{2}} r^{2}\right)^{2} \text { ExpIntegralEi }\left[1+2 A r^{2}\right]\right] \text {. }
\end{aligned}
$$

\subsection{New Class I anisotropic solution generated via MGD} approach for two systems with same complexity factor

In this section, we will investigate a new Class I anisotropic solution via MGD approach for which the two systems have same complexity factor. This can be done by assuming $\hat{Y}_{T F}=Y_{T F}$ which means the complexity factor $Y_{T F}$ related to energy-momentum tensor $T_{i j}$ does not change after the gravitational decoupling. This condition gives $Y_{T F}^{\Theta}=0$ and can be expanded as

$$
\Pi_{\Theta}=\frac{1}{2 r^{3}} \int_{0}^{r} x^{3}\left[\Theta_{0}^{0}(x)\right]^{\prime} d x,
$$

where we can write the right side integral of above Eq. (69) by using Eq. (27) as,

$$
\int_{0}^{r} x^{3}\left[\Theta_{0}^{0}(x)\right]^{\prime} d x=\frac{r^{3}}{4 \pi}\left(\frac{f}{r^{2}}-\frac{f^{\prime}}{2 r}\right) .
$$

Then using the Eqs. (69) and (70) together with Eqs. (28)(30), we get

$f^{\prime}\left(4 r+\xi^{\prime} r^{2}\right)+f\left(2 \xi^{\prime \prime} r^{2}+\xi^{\prime 2} r^{2}-8-2 \xi^{\prime} r\right)=0$.

We observe here that the solution of equation (71) again depends on the solution of the seed spacetime (43) described by the metric functions $\xi$ and $\mu$. Therefore, we again use the same Class I spacetime geometry (38) for solving this differential equation (71),

$e^{\xi(r)}=B e^{2 A r^{2}}, \quad$ and $\quad \mu(r)=\frac{1}{1+D A r^{2} e^{2 A r^{2}}}$.

and corresponding complexity factor the $Y_{T F}$ generated by the above Class I solution (72) is determined by using the definition (32) as,

$Y_{T F}=\frac{2 A^{2}\left(2-3 D e^{2 A r^{2}}+D^{2} e^{4 A r^{2}}\right) r^{2}}{\left(1+A D e^{2 A r^{2}} r^{2}\right)^{2}}$, 
Fig. 3 The behavior of radial pressure $\left(P_{r} \times 10^{4}\right)$-top left, tangential pressures $\left(P_{\perp} \times 10^{4}\right)$-top right, energy density $\left(\epsilon \times 10^{4}\right)$-bottom left and anisotropy

$\left(\hat{\Pi} \times 10^{4}\right)$-bottom right versus radial coordinate $r / R$ for different coupling constant $\beta$ with compactness factor $\frac{M_{s}}{R}=0.25$. The above figures are plotted for the solution 4.1 corresponding to the case $\hat{\Pi}=0$
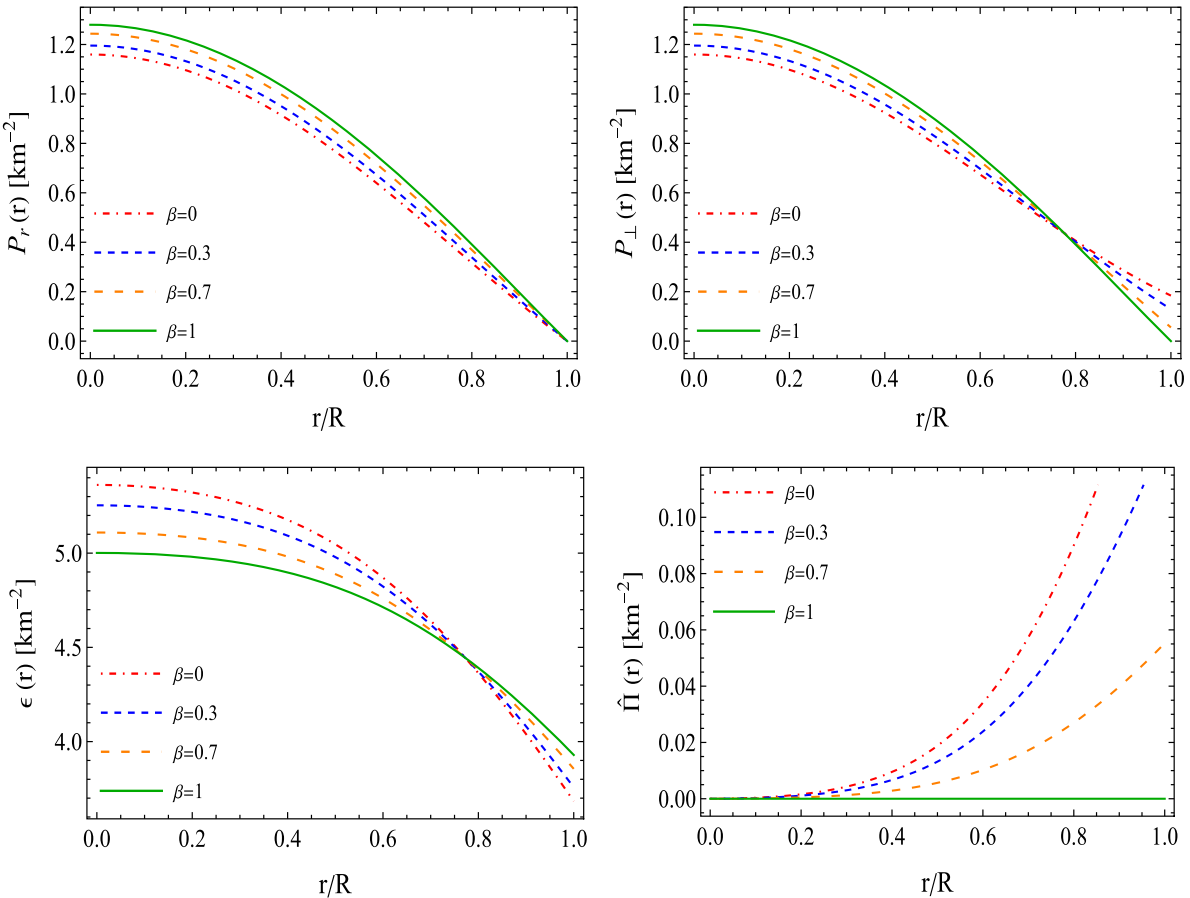

By plugging the spacetime geometry (72) into the Eq. (70), we find the deformation function $f(r)$ as,

$f(r)=F e^{-2 A r^{2}} r^{2}\left(1+A r^{2}\right)$,

where $F$ is a constant of integration with dimension length $^{-2}$ and this deformation function $f(r)$ gives condition $\hat{Y}_{T F}=$ $Y_{T F}$ for which $Y_{T F}^{\Theta}=0$. However, the new version of Class I metric functions can be read as,

$e^{-\lambda(r)}=\frac{1}{1+D A r^{2} e^{2 A r^{2}}}+\frac{\beta F r^{2}}{e^{2 A r^{2}}}\left(1+A r^{2}\right)$,

$e^{v(r)}=B e^{2 A r^{2}}$.

The above solution (75)-(76) gives the effective pressures and energy density,

$$
\begin{aligned}
& P_{r}(r, \beta, F)=\frac{\beta F}{e^{2 A r^{2}}}\left(1+A r^{2}\right)\left(1+4 A r^{2}\right) \\
& -\frac{A\left(D e^{2 A r^{2}}-4\right)}{1+A D e^{2 A r^{2}} r^{2}}, \\
& P_{\perp}(r, \beta, F) \\
& =\frac{A\left(4-D e^{2 A r^{2}}+4 A r^{2}\right)}{\left(1+A D e^{2 A r^{2}} r^{2}\right)^{2}}+\frac{\beta F}{e^{2 A r^{2}}}\left(1+6 A r^{2}\right. \\
& \left.\quad+6 A^{2} r^{4}\right), \\
& \epsilon(r, \beta, F)=\frac{A D\left(3+A\left(4+D e^{2 A r^{2}}\right) r^{2}\right)}{e^{-2 A r^{2}}\left(1+A D e^{2 A r^{2}} r^{2}\right)^{2}} \\
& +\frac{\beta F,}{e^{2 A r^{2}}}\left(4 A^{2} r^{4}-3-A r^{2}\right),
\end{aligned}
$$

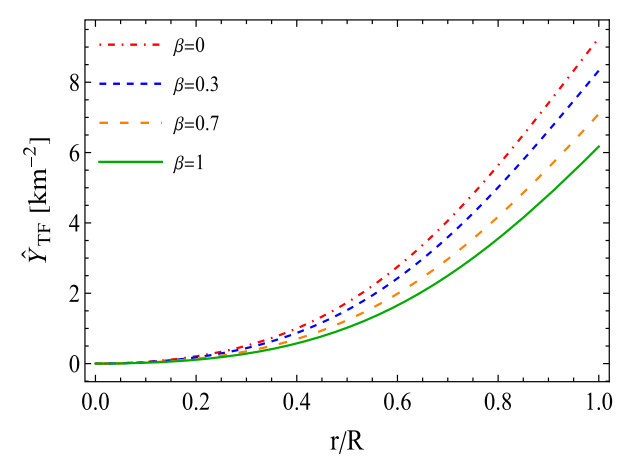

Fig. 4 The behavior of the complexity factor $\left(\hat{Y}_{T F} \times 10^{4}\right)$ versus radial coordinate $r / R$ for different coupling constant $\beta$ with compactness factor $\frac{M_{s}}{R}=0.25$. This complexity figure is plotted for the minimally deformed Class I solution 4.1

and effective anisotropy $\hat{\Pi}=P_{\perp}-P_{r}$ is

$$
\begin{aligned}
\hat{\Pi}(r, \beta, F)= & \frac{A\left(4-D e^{2 A r^{2}}+4 A r^{2}\right)}{\left(1+A D e^{2 A r^{2}} r^{2}\right)^{2}}+\frac{\beta F}{e^{2 A r^{2}}} A r^{2} \\
& \times\left(1+2 A r^{2}\right)+\frac{A\left(-4+D e^{2 A r^{2}}\right)}{1+A D e^{2 A r^{2}} r^{2}} .
\end{aligned}
$$

We would like to comment here that the gravitational potentials (75) and (76) and matter variables described by Eqs. (77)-(80) represent a new version of Class I anisotropic solutions (43) corresponding to the Einstein field equations (7)-(9), whose complexity factor $\hat{Y}_{T F}$ is the same as the complexity factor $Y_{T F}$ given by Eq. (73). On the other hand, applying the matching conditions (48)-(50) for the new solu- 


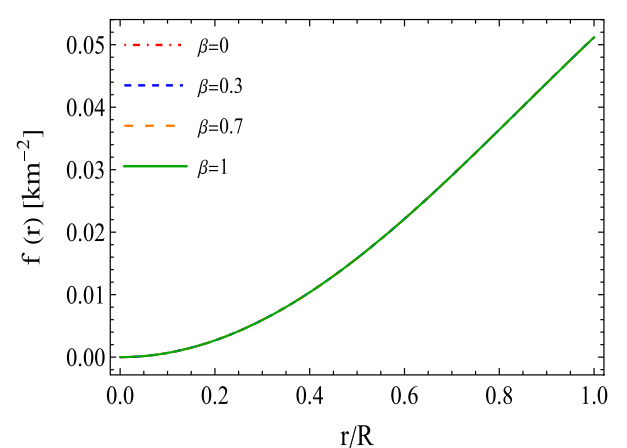

Fig. 5 The behavior of the deformation function versus radial coordinate $r / R$ for the solution 4.2 corresponding to the case $\hat{Y}_{T F}=Y_{T F}$

tion (74)-(80) determines the modified form of the constant parameters $D$ and $B$, and $M$ as,

$$
\begin{aligned}
D & =\frac{e^{-2 A R^{2}}\left[4 A^{2} \beta F R^{4}+5 A \beta F R^{2}+4 A e^{2 A R^{2}}+\beta F\right]}{A\left[e^{2 A R^{2}}-\beta F R^{2}\left(4 A^{2} R^{4}+5 A R^{2}+1\right)\right]}, \\
B & =\ln \left[\frac{1}{1+D A R^{2} e^{2 A R^{2}}}+\frac{\beta F R^{2}}{e^{2 A R^{2}}}\left(1+A R^{2}\right)\right]-2 A R^{2}, \\
M & =M_{s}-\frac{\beta F R^{3}}{2 e^{2 A R^{2}}}\left(1+A R^{2}\right) .
\end{aligned}
$$

where $M_{s}=\frac{R}{2}(1-\mu)$ and therefore under new constant $D \longrightarrow D_{\beta F}$ (mentioned by Eq. 81), the complexity factor $\hat{Y}_{T F}$ will become,

$\hat{Y}_{T F}=\frac{2 A^{2}\left(2-3 D_{\beta F} e^{2 A r^{2}}+D_{\beta F}^{2} e^{4 A r^{2}}\right) r^{2}}{\left(1+A D_{\beta F} e^{2 A r^{2}} r^{2}\right)^{2}}$
Here, we can see the effect of the $\beta$ on the complexity factor due to the modified constant value of $D$ but the total complexity factor will be same.

\section{Some physical properties of the solutions}

In this section, we present a physical analysis of the solutions obtained in Sect. 4 based on the trends of the plots in order to test their viability:

For solution 4.1: Figure 2 shows that the deformation function $f(r)$ is positive for $0<r<R$ but it is zero at the center as well as the boundary of the star. This implies that the total mass $M$ of the stellar object for the gravitationally decoupled solution remains the same as $M_{s}$ even after introducing the MGD. In this situation, the gravitational mass is distributed only inside the stellar object. In Fig. 3, we show the trend for radial and tangential pressures, energy density, and anisotropy for the MGD Class I solution within the stellar object. It is observed that $P_{r}, P_{\perp}$, and $\epsilon$ are decreasing monotonically towards the surface for all values of $\beta=0,0.3,0.7$, and 1 . The central pressures and surface density are increasing when $\beta$ increases while central density has the opposite behavior. The anisotropy is increasing away from the center when $\beta=0,0.3,0.7$ but it vanishes throughout the model for $\beta=1$ (green curve). The vanishing of effective anisotropy throughout the model represents a Class I isotropic solution corresponding to matter distribution $\hat{T}_{i j}$ i.e. $P_{r}=P_{\perp}$ for all $r \in[0, R]$ which is clearly observed by the green curves in the top panel of this figure. More-
Fig. 6 The behavior of radial pressure $\left(P_{r} \times 10^{4}\right)$-top left, tangential pressures $\left(P_{\perp} \times 10^{4}\right)$-top right, energy density $\left(\epsilon \times 10^{4}\right)$-bottom left and anisotropy

$\left(\hat{\Pi} \times 10^{4}\right)$-bottom right versus radial coordinate $r / R$ for different coupling constant $\beta$ with compactness factor $\frac{M_{s}}{R}=0.25$ with $F=0.0002$. The above figures are plotted for the anisotropic solution 4.2 corresponding to the case $\hat{Y}_{T F}=Y_{T F}$
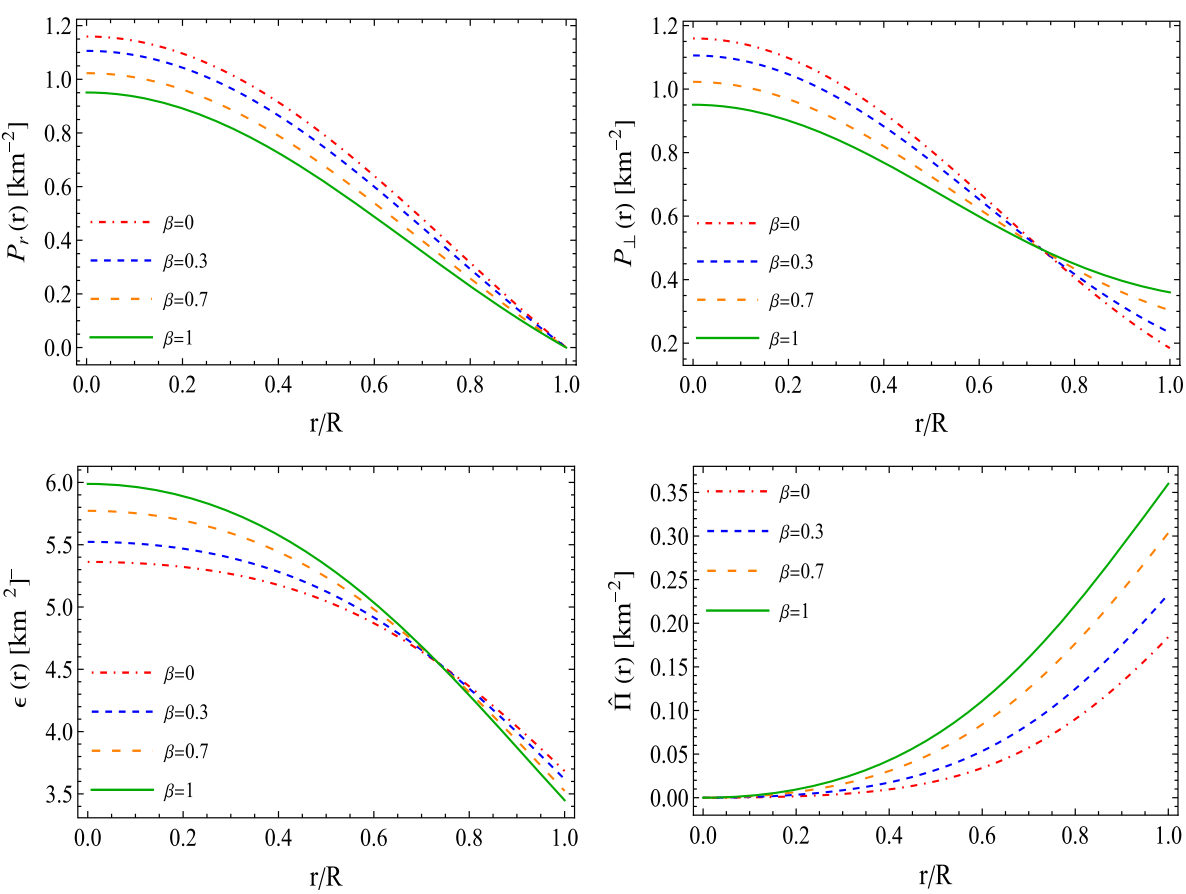


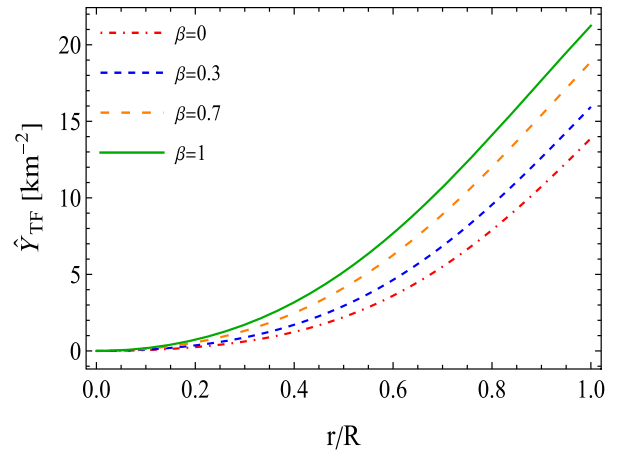

Fig. 7 The behavior complexity factor $\hat{Y}_{T F}$ versus radial coordinate $r / R$ for different decoupling constant $\beta$ with compactness factor $\frac{M_{s}}{R}=0.25$ and $F=0.0005$. The above complexity figure is plotted for solution 4.2 corresponding to the case $\hat{Y}_{T F}=Y_{T F}$

over, the variation of the complexity factor for this solution is mentioned in Fig. 4 for different values of $\beta$. It is observed that the complexity factor is increasing towards the boundary but its value decreases as the decoupling constant $\beta$ is increased. This implies that the impact of complexity is less in the context of MGD.

For solution 4.2: Here, the situation is different than solution 4.1. As we can see in Fig. 5 that the deformation function is increasing and non-negative throughout the model as well as attaining a non-zero value at the boundary. In this case, the total mass $(M)$ of the gravitationally decoupled object will be less than the total mass $M_{s}$ of the object in pure GR due to positive $\beta$. However, the effective pressures and density for this solution show the same behavior as shown in solution 4.1 which is decreasing throughout the stellar object, but the magnitude values of pressure and density show totally opposite behavior as compared to solution 4.1. Moreover, the effective anisotropy $(\hat{\Pi})$ is monotonically increasing from center to boundary for all $\beta$ which implies the anisotropic force acting in outward direction and value of $\hat{\Pi}$ increases when $\beta$ moves from 0 to 1 (see Fig. 6). Furthermore, we also check the behavior of complexity factors for this solution 4.2 which is increasing within the objects (Fig. 7). It is also observed that the values of the complexity factor increases when $\beta$ increase which shows an opposite trend as obtained in solution 4.1.

\section{Concluding remarks}

In the present work, we have investigated a new isotropic Class I solution in the framework of gravitational decoupling through using a minimal geometric deformation approach. To do this, we use the isotropy condition for determining the deformation function $f(r)$ under which the gravitationally decoupled system becomes isotropic. The viability of the obtained isotropic Class I solution has been tested through the variations of physical quantities such as pressure, density and anisotropy within the stellar object. All the physical quantities of the solution satisfy the conditions for a wellbehaved stellar model. Therefore, we can say that the MGD approach has equipped us with a novel method to generate new physically viable Class I isotropic solutions. On the other hand, we use another alternative approach [60], which are the two systems with same complexity factors, in order to find the deformation function $f(r)$. This is also one of the effective approaches to find a new well-behaved solution or generalized previous known solutions in the context of gravitational decoupling. The idea of complexity was well studied by Herrera and co-workers within the framework of dissipative, radiating collapse. They showed that a spherically symmetric, shear-free star undergoing dissipative collapse while radiating energy in the form of a radial heat flux could mimic shear-like effects arising from pressure anisotropy, density inhomogeneity and heat flow [63-65]. Furthermore, Herrera [66] demonstrated that the isotropy condition is unstable, ie., a self-gravitating body with isotropic pressure (radial and tangential stresses equal at each interior point) in quasi-static equilibrium will evolve into an anisotropic distribution as collapse proceeds. There have been numerous studies on the dissipative collapse of radiating stars starting off from an initial static configuration [67] but these models lacked any mechanism which could explain the onset of any instabilities driving them away from hydrostatic equilibrium. The models that we presented here could describe the onset of dissipative collapse arising from the anisotropisation of the radial and tangential stresses within the initial static configuration or the isotropisation of the pressure when the collapse leads to a final static star in hydrostatic equilibrium $[68,69]$.

Acknowledgements The author SKM is thankful for continuous support and encouragement from the administration of University of Nizwa. Also, SKM acknowledges that this work is supported by TRC Project (Grant No. BFP/RGP/CBS-/19/099), the Sultanate of Oman.

Data Availability Statement This manuscript has no associated data or the data will not be deposited. [Authors' comment: This is a theoretical study and the results can be verified from the information available.]

Open Access This article is licensed under a Creative Commons Attribution 4.0 International License, which permits use, sharing, adaptation, distribution and reproduction in any medium or format, as long as you give appropriate credit to the original author(s) and the source, provide a link to the Creative Commons licence, and indicate if changes were made. The images or other third party material in this article are included in the article's Creative Commons licence, unless indicated otherwise in a credit line to the material. If material is not included in the article's Creative Commons licence and your intended use is not permitted by statutory regulation or exceeds the permitted use, you will need to obtain permission directly from the copyright holder. To view a copy of this licence, visit http://creativecomm ons.org/licenses/by/4.0/.

Funded by $\mathrm{SCOAP}^{3}$. 


\section{Appendix}

$$
\begin{aligned}
& \Psi_{1}(r)=e^{-2 A r^{2}} \\
& \times\left(F-\frac{2 A \text { ExpIntegralEi }\left[1+2 A r^{2}\right]}{e}+\frac{A D e^{4 A r^{2}}}{\left(1+A D e^{\left.2 A r^{2} r^{2}\right)}\right.}\right) \\
& \Psi_{2}(r)=-\frac{2 e^{-1-2 A r^{2}}}{\left(1+2 A r^{2}\right)\left(1+A D e^{\left.2 A r^{2} r^{2}\right)^{2}}\right.} \\
& \quad \times\left[e \left\{F\left(1+A D e^{2 A r^{2}} r^{2}\right)^{2}\left(-1+4 A^{2} r^{4}\right)\right.\right. \\
& +A e^{2 A r^{2}}\left(4 A r^{2}+4 A^{3} D^{2} e^{4 A r^{2}} r^{6}\right. \\
& \left.\left.+D e^{2 A r^{2}}\left(-1-4 A r^{2}+4 A^{2} r^{4}\right)\right)\right\} \\
& -2 A\left(1+A D e^{2 A r^{2}} r^{2}\right)^{2} \\
& \left.\times\left(-1+4 A^{2} r^{4}\right) \operatorname{ExpIntegralEi}^{2}\left[1+2 A r^{2}\right]\right]
\end{aligned}
$$

\section{References}

1. S. W. Israel, W. Israel, 300 Years of Gravitation, Cambridge University Press (1989)

2. S. Weinberg, Gravitation and Cosmology (John Wiley, Singapore, 2004)

3. B.P. Abbott et al., Phys. Rev. Lett. 116, 6 (2016)

4. R. S. Park, W. M. Folkner, A. S. Konopliv1, J. G. Williams, D. E. Smith, M. T. Zuber, Astron. J. 2017153121

5. The Event Horizon Telescope Collaboration, Astrophys. J. Lett. 875, L1 (2019)

6. R. Durrer, R. Maartens, Gen. Relativ. Gravit. 40, 301 (2007)

7. I. Debono, G.F. Smoot, Universe 2, 23 (2016)

8. K. Schwarzschild, Sitz. Deut. Akad. Wiss. Berlin. Kl. Math. Phys. 1, 189 (1916)

9. M.S.R. Delgaty, K. Lake, Comput. Phys. Comm. 115, 395 (1998)

10. K. Dev, M. Gleiser, Gen. Relativ. Gravit. 34, 1793 (2002)

11. K. Dev, M. Gleiser, Gen. Relativ. Gravit. 35, 1435 (2003)

12. R. Penrose, Riv. Nuovo Cimento Soc. Ital. Fis. 1, 252 (1969)

13. J.R. Oppenheimer, H. Snyder, Phys. Rev. 56, 455 (1939)

14. C.H. Brans, R.H. Dicke, Phys. Rev. 124, 925 (1961)

15. A.A. Starobinsky, JETP Lett. 86, 157 (2007)

16. D. Lovelock, J. Math. Phys. 12, 498 (1971)

17. D. Lovelock, J. Math. Phys. 13, 874 (1972)

18. T. Harko, F.S.N. Lobo, S. Nojiri, S.D. Odintsov, Phys. Rev. D 84, 024020 (2011)

19. P. Rastall, Phys. Rev. D 6, 3357 (1972)

20. N.F. Naidu, R.S. Bogadi, A. Kaisavelu, M. Govender, Gen. Relativ. Gravit. 52, 79 (2020)

21. J. Ovalle, Phys. Rev. D 95, 104019 (2017)

22. J. Ovalle, Phys. Lett. B 788, 213 (2019)

23. S. K. Maurya, K. N. Singh, M. Govender, S. Hansraj, arXiv:2109.00358v2 [gr-qc] (2021)

24. J. Ovalle, R. Casadio, R. Da Rocha, A. Sotomayor, Z. Stuchlik, EPL 124, 20004 (2018)

25. G. Panotopoulos, A. Rincón, Eur. Phys. J. C 78, 851 (2018)

26. G. Abbas, H. Nazar, Eur. Phys. J. C 78, 510 (2018)
27. C. Las Heras, P. León, Fortsch. Phys. 66, 1800036 (2018)

28. L. Gabbanelli, A. Rincón, C. Rubio, Eur. Phys. J. C 78, 370 (2018)

29. S. Hensh, Z. Stuchlk, Eur. Phys. J. C 79, 834 (2019)

30. J. Ovalle, R. Casadio, R. da Rocha, A. Sotomayor, Eur. Phys. J. C 78, 122 (2018)

31. J. Ovalle, A. Sotomayor, Eur. Phys. J. Plus 133, 428 (2018)

32. J. Ovalle, R. Casadio, R. da Rocha, A. Sotomayor, Z. Stuchlik, Eur. Phys. J. C 78, 960 (2018)

33. E. Contreras, A. Rincón, P. Bargueño, Eur. Phys. J. C 79, 216 (2019)

34. C. Las Heras, P. León, Eur. Phys. J. C 79, 990 (2019)

35. J. Ovalle, C. Posada, Z. Stuchlik, Class. Quantum Gravity 36(20), 205010 (2019)

36. M. Estrada, R. Prado, Eur. Phys. J. Plus 134, 168 (2019)

37. M. Estrada, Eur. Phys. J. C 79, 918 (2019)

38. S.K. Maurya, Eur. Phys. J. C 80, 429 (2020)

39. S.K. Maurya, K.N. Singh, B. Dayanandan, Eur. Phys. J. C 80, 448 (2020)

40. M. Sharif, Q. Ama-Tul-Mughani, Ann. Phys. 415, 168122 (2020)

41. M. Zubair, H. Azmat, Ann. Phys. 420, 168248 (2020)

42. P. Leon, A. Sotomayor, Fortschr. Phys. 69, 2100017 (2021)

43. H. Azmat, M. Zubair, Eur. Phys. J. C Plus 136, 112 (2021)

44. E. Contreras, E. Fuenmayor, Phys. Rev. D 103, 124065 (2021)

45. S.K. Maurya, A.M. Al Aamri, A.K. Al Aamri, R. Nag, Eur. Phys. J 81, 701 (2021)

46. M. Zubair, M. Amin, H. Azmat, Phys. Scripta 96, 125008 (2021)

47. Q. Muneer, M. Zubair, M. Rahseed, Phys. Scripta 96, 125015 (2021)

48. M. Zubair, H. Azmat, M. Amin, Chin. J. Phys. (2021). https://doi. org/10.1016/j.cjph.2021.07.035

49. E. Contreras, J. Ovalle, R. Casadio, Phys. Rev. D 103, 044020 (2021)

50. J. Ovalle, R. Casadio, E. Contreras, A. Sotomayor, Phys. Dark Univ. 31, 100744 (2021)

51. S.K. Maurya, A. Pradhan, F. Tello-Ortiz, A. Banerjee, R. Nag, Eur. Phys. J. C 81, 848 (2021)

52. S.K. Maurya, F. Tello-Ortiz, M. Govender, Fortschr. Phys. 69, $2100099(2021)$

53. S.K. Maurya, L.S. Said Al-Farsi, Eur. Phys. J 136, 317 (2021)

54. L. Herrera, Phys. Rev. D 97, 044010 (2018)

55. L. Herrera, A. Di Prisco, J. Ospino, Phys. Rev. D 98, 104059 (2018)

56. L. Herrera, A. Di Prisco, J. Carot, Phys. Rev. D 99, 124028 (2019)

57. M. Carrasco-Hidalgo, E. Contreras, Eur. Phys. J. C 81, 757 (2021)

58. J. Andrade, E. Contreras, Eur. Phys. J. C 81, 889 (2021)

59. C. Arias, E. Contreras, E. Fuenmayor, A. Ramos, Ann. Phys. 436, 168671 (2022)

60. R. Casadio, E. Contreras, J. Ovalle, A. Sotomayor, Z. Stuchlick, Eur. Phys. J. C 79, 826 (2019)

61. R. Tolman, Phys. Rev. 35, 875 (1930)

62. L.P. Eisenhart, Riemannian Geometry (Princeton University Press, Princeton, NJ, p. 97, 1925)

63. L. Herrera, A. Di Prisco, J. Hernandez-Pastora, N.O. Santos, Phys. Lett. A 237, 113 (1998)

64. L. Herrera, A. Di Prisco, J. Ospino, Gen. Relativ. Gravit. 42, 1585 (2010)

65. L. Herrera, Int. J. Mod. Phys. D 20, 1689 (2011)

66. L. Herrera, Phys. Rev. D 101, 104024 (2020)

67. W.B. Bonnor, A.K.G. de Oliveira, N.O. Santos, Phys. Rep. 181, 269 (1989)

68. M. Govender, K.S. Govinder, S.D. Maharaj, R. Sharma, S. Mukherjee, T.K. Dey, Int. J. Mod. Phys. D 12, 667 (2003)

69. M. Govender, W. Govender, K.P. Reddy, S.D. Maharaj, Eur. Phys. J. C 81, 177 (2021) 